\title{
Orthorexia nervosa
}

\section{Kann gesunde Ernährung eine Krankheit sein?}

\begin{abstract}
Angesichts der aktuellen Lebensmittelskandale schauen wohl die meisten Menschen etwas genauer, was auf ihrem Teller landet. Das Bedürfnis nach unbelastetem und gesundem Essen kann aber auch zur Obsession werden. Wann eine sogenannte Orthorexia nervosa besteht, erklärt die Psychologin Friederike Barthels von der Heinrich-Heine-Universität Düsseldorf.
\end{abstract}

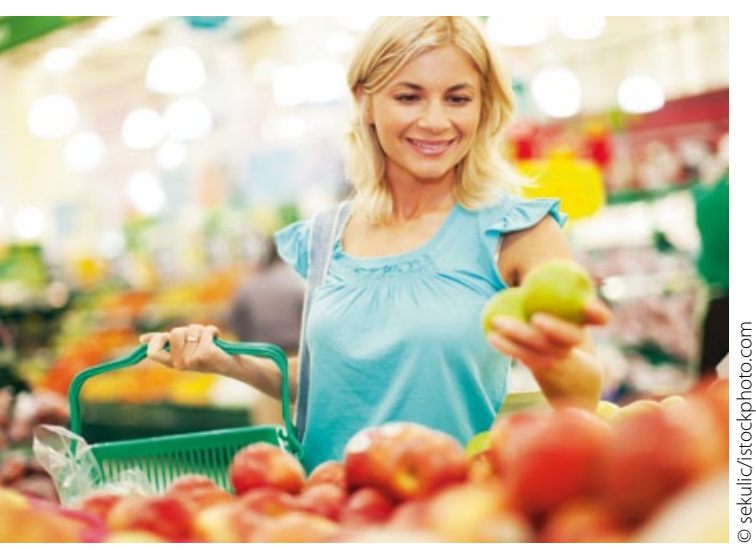

Sind diese Äpfel auch wirklich gesund?

MMW: Wie sieht der Speiseplan eines Orthorektikers aus?

Barthels: Das hängt stark davon ab, was der Betroffene für sich selbst als gesunde Ernährungsweise definiert hat. Manche kaufen nur Bioprodukte vom Bauernhof, andere vermeiden Zusatzstoffe wie Farbstoffe oder Geschmacksverstärker. Es gibt aber auch Betroffene, die auf bestimmte Lebensmittel oder Lebensmittelgruppen und sogar auf essenzielle Inhaltsstoffe verzichten, die z. B. Fett oder Kohlenhydrate für gesundheitsschädlich halten.

MMW: Wann ist denn die Grenze von einer gesundheitsbewussten Ernährung zur Orthorexia nervosa überschritten?

Barthels: Die Grenze ist überschritten, wenn jemand einen Leidensdruck entwickelt. Das passiert, wenn gesunde Ernährung zum Zwang wird und jemanden den ganzen Tag beschäftigt, wenn der Betroffene nicht mehr überall einkaufen kann oder Einladungen ausschlägt, weil er Angst hat, mit ungesunden Nahrungsmitteln konfrontiert zu werden. In Extremfällen, wenn die Ernährung sehr ein- seitig ausfällt, kann Orthorexie zu Mangelerscheinungen und Abmagerung führen.

MMW: Sie haben im Team von Professor Pietrowsky einen Fragebogen für die Orthorexie entwickelt. Was sind die entscheidenden Kriterien?

Barthels: Eine Frage ist z. B., ob jemand Ernährungsregeln aufgestellt hat, die er sehr streng und rigide befolgt. Die Auswahl von Nahrungsmitteln richtet sich stärker nach gesundheitlichen Aspekten als nach dem Genuss beim Essen. Die Gedanken kreisen ständig um die gesunde Ernährung, sodass wenig Zeit bleibt für Hobbys und Freunde und manchmal auch die Arbeit darunter leidet.

MMW: Wird die Orthorexia nervosa als eigenständiges Krankheitsbild angesehen? Barthels: Das erforschen wir noch. In mancher Hinsicht gibt es Überschneidungen mit der Anorexia nervosa, etwa beim Verzicht auf bestimmte Nahrungsmittel oder bei Essensritualen. Ich persönlich glaube, dass die Orthorexie etwas Eigenes ist, weil hier der Gesundheitsgehalt der Nahrung im Mittelpunkt steht und nicht die Kalorienzahl. Der Krankheitsbegriff ist aber auch deshalb schwierig, weil fraglich ist, inwieweit die Betroffenen wirklich leiden. Viele sind sehr überzeugt von ihrer Ernährungsweise und ihrem Lebensstil.

MMW: Wie häufig tritt Orthorexie auf? Barthels: Wir haben Online-Umfragen mit unserer Orthorexie-Skala durchgeführt. Danach hatten etwa 1-2\% der Befragten extrem hohe Punktzahlen, was ein Hinweis auf das Vorliegen einer Orthorexie sein kann. Frauen und jüngere Erwachsene zwischen 20 und 40 Jahren waren etwas öfter betroffen.

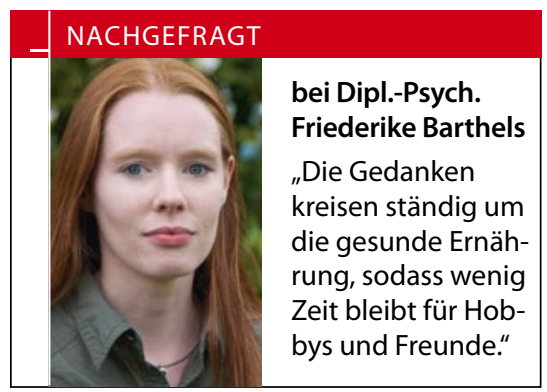

MMW: Wann sollte man als Arzt Verdacht schöpfen, dass eine gesundheitsbewusste Ernährung in orthorektisches Essverhalten umschlägt?

Barthels: Man sollte hellhörig werden, wenn man das Gefühl hat, dass ein Patient nur noch seine Ernährung im Kopf hat. Das gilt besonders, wenn er über eine extrem einseitige Ernährung berichtet, z. B. dass er komplett auf Fett verzichtet, und natürlich wenn Mangelerscheinungen auftreten. Besonders aufmerksam muss man sein, wenn Kinder im Spiel sind, da es gefährlich werden kann, wenn orthorektische Eltern ihre Kinder genauso extrem ernähren wie sich selbst.

MMW: Jemanden von einem Verhalten zu kurieren, dem er sich um seiner Gesundheit willen unterzieht, ist vermutlich schwierig. Wie kann man den Betroffenen helfen? Barthels: Wir können noch nicht genau sagen, welche therapeutischen Angebote sinnvoll sind. Wichtig wäre es aufzuzeigen, dass es nicht nur eine einzige Form gesunder Ernährung gibt, sondern dass es immer auf die Kombination und die Menge ankommt - dass etwa der Verzehr von einem Stück Schokolade kein Verbrechen ist. Wenn das alles nichts bringt, weil die Betroffenen zu sehr von ihren Vorstellungen eingenommen sind, kann man über eine Verhaltenstherapie nachdenken.

- Interview: Dr. Beate Schumacher

\section{Lesetipp}

Bei aller Besorgnis um eine allzu gesundheitsfixierte Ernährungsweise - für die ganz überwiegende Mehrzahl der Patienten ist ungesundes, überreichliches Essen das Hauptproblem. Welche Diäten wirklich Erfolg versprechen, ist Thema unseres Schwerpunktes in dieser MMW ab S. 44 . 